\title{
ESTUDIOS FENOMORFOLÓGICOS EN LA VEGETACIÓN DEL SUR DE ESPAÑA. I. CISTUS L.
}

\author{
Baltasar CABEZUDO, Teresa NAVARRO, Andrés V. PÉREZ LATORRE, \\ José M. NIETO CALDERA y Gideon ORSHAN
}

\begin{abstract}
RESUMEN. Se describe el comportamiento fenomorfológico de cinco especies de Cistus L. (C. ladanifer L., C. populifolius L. subsp. major (Pourret ex Dunal) Heywood, C. albidus L., C. monspeliensis L. y C. salvifolius L.) presentes en la serie de los alcornocales con encinas del sector Malacitano-Axarquiense (Málaga, España). Se discute el comportamiento fenológico y se comparan los resultados con los ya obtenidos en otras zonas del área mediterránea.
\end{abstract}

Palabras clave. Fenomorfología, Cistus, España.

\begin{abstract}
The phenomorphology of five Cistus species was studied in the Montes de Málaga range (Málaga) of southern Spain. The study sites was visited at least once every month during a whole year. Each of the species was carefully examined, described in the field and representative herbarium specimens collected to form a supporting phenological herbarium. Pheno-morphological diagrams were then made describing the annual cycles of the plants. For three species the diagrams were compared to similar ones made in France and for one to a diagram made also in Israel.

The phenophases of flower bud formation, of flowering, of fruit setting, of seed dispersal and of dolichoblast leaf shedding were similar for the species examined in Spain and also in France and Israel. Since no distinction was made between brachyblasts of the current year to those of preceeding ones more variation between species and countries was obtained.
\end{abstract}

Key words. Pheno-morphology, Cistus, Spain.

\section{INTRODUCCIÓN}

Son escasos los estudios fenomorfológicos que describen los cambios estacionales de órganos y morfología de las plantas durante su ciclo completo de vida en la región Mediterránea (Orshan 1964, Hoffmann 1972, Hoffmann y Hoffmann 1976, Montenegro et al., 1979, Jow et al., 1980, Hoffmann y Walker 1980, Aljaro et al., 1981, Westman 1981, Hoffmann 1981, Rutherford y Panagos 1982, Orshan 1989). Estos estudios permiten detectar el origen, crecimiento, senescencia y desprendimiento de cada parte de una planta, hojas, ramas e inflorescencias, así como conocer mejor su estructura y ciclo anual. La relación de las fenofases de cada órgano con los cambios estacionales del medio nos permite obtener un mejor conocimiento de las relaciones planta-ambiente. Estos estudios fenomorfológicos se han iniciado en Andalucía, centrándose fundamentalmente en formaciones caracterizadas por el alcornoque

Trabajo realizado gracias a la ayuda concedida por la Junta de Andalucía al Grupo de Investigación nº 0413 de la Universidad de Málaga. 
(Quercus suber L.). Damos a conocer en este primer trabajo los datos referentes a diversas especies del género Cistus L.

El estudio fue realizado en Málaga (Venta Lanada, Montes de Málaga, coord. U.

T. M. 30SUF8078). La zona elegida se encuentra a una altitud de 830 $890 \mathrm{~m}$. s. m. con fuertes pendientes orientadas al norte. La litología dominante está formada por esquistos, cuarcitas y areniscas. Los suelos corresponden a tierra parda forestal bajo vegetación clímax pero se erosionan y se hacen superficiales tras la destrucción del bosque. Bioclimáticamente el área se incluye en el piso mesomediterráneo con ombroclima subhúmedo (Rivas Martínez, 1987). La temperatura media anual ronda los $13^{\circ} \mathrm{C}$. La precipitación media anual es de unos 700 mm. con 3-4 meses de sequía veraniegos (figura 1 y tabla 1 ).

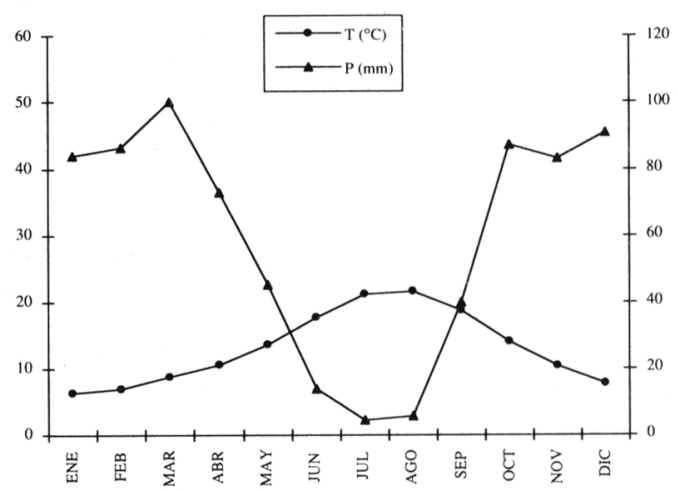

Figura 1. Diagrama ombrotérmico.

\begin{tabular}{lccccrrrrrr}
\hline Estación & P & T & It & Ia & ETP & BA & EST & PF & PS & K \\
\hline Benefique & 744 & 12.6 & 231 & 33.9 & 696 & +48 & IOPV & $3-4$ & 3 & 23.6 \\
Torrijos & 788 & 13.8 & 281 & 33.1 & - & - & - & - & - & - \\
Contadoras & 682 & 13.7 & 285 & 28.8 & 728 & -46 & IOPV & $3-4$ & 3.5 & 21.9 \\
\hline
\end{tabular}

Tabla 1. Parámetros climáticos de la zona estudiada. $\mathrm{P}=$ precipitación media anual, $\mathrm{T}=$ temperatura media anual, $\mathrm{It}=$ índice de termicidad de Rivas Martínez, Ia=índice de aridez de De Martonne, ETP=evapotranspiración anual, BA=P-ETP, EST=estacionalidad de las precipitaciones (IOPV=Invierno mayor que Otoño mayor que Primavera mayor que Verano), $\mathrm{PF}=$ período frío de Emberger, $\mathrm{PS}=$ período seco, $\mathrm{K}=$ =índice de continentalidad de Gorezinsky. (datos procedentes de De León Llamazares 1989 y Rivas Martínez, 1987).

La vegetación potencial de la zona es incluíble en la serie de los alcornocales con encinas (Teucrio-Querceto suberis quercetoso rotundifoliae sigmetum) del sector Malacitano-Axarquiense (Nieto Caldera et als, 1991). Actualmente sólo es posible hallar de modo disperso restos de los bosques de Quercus suber ya que la zona ha sido fuertemente transformada por actividades humanas que han dado como resultado la presencia de numerosos cultivos marginales y matorrales de sustitución (jarales).

\section{MATERIAL Y MÉTODOS}

Las cinco especies de Cistus estudiadas son: C. ladanifer L., C. populifolius L. subsp. major (Pourret ex Dunal) Heywood, $C$. albidus L., C. monspeliensis L. y $C$. 
salvifolius L. (Warburg, 1968). Dos de las especies, $C$. populifolius subsp. major y $C$. ladanifer, son estrictamente mediterráneo-occidentales; la primera aparece en la Península Ibérica, Francia y Marruecos mientras que la segunda también se encuentra en dichas regiones y Argelia. La distribución de $C$. albidus es más extensa alcanzando Italia y Túnez por el este y ocupando también las islas de oeste del Mediterráneo. $C$. monspeliensis y $C$. salvifolius se encuentran prácticamente en todo el Mediterráneo y se extienden hacia el norte de Europa e Irán. (Greuter et al., 1984).

El método utilizado está descrito en detalle por Orshan (1989). Cada especie fue descrita en el campo durante un año (Septiembre de 1990 a Octubre de 1991), siendo la periodicidad del estudio una vez cada cuatro o tres semanas. Se puso especial atención a los distintos tipos de ramificaciones, hojas, inflorescencias, flores y frutos. La idea era obtener un conocimiento cualitativo del desarrollo estacional y reemplazamiento de las partes de la planta durante el año, por lo que sus estados de desarrollo fueron recogidos sin medir y determinar dimensiones de las partes de la planta. En algunos casos fue necesario estimar (no medir) valores medios de las dimensiones de partes de la planta.

En cada muestreo se recolectaron partes representativas de cada especie realizándose con ellas un herbario fenomorfológico; los pliegos del herbario demostraron ser una ayuda inestimable para realizar los diagramas fenomorfológicos, ya que algunas fases u órganos difícilmente detectables en el campo fueron mejor estudiados en el material del herbario.

Los siguientes símbolos, que representan los órganos de la planta y las fases fenológicas en los diagramas fenomorfológicos, están estandarizados (Orshan, 1989) y son los utilizados para todas las especies examinadas:

A. Órganos de la planta
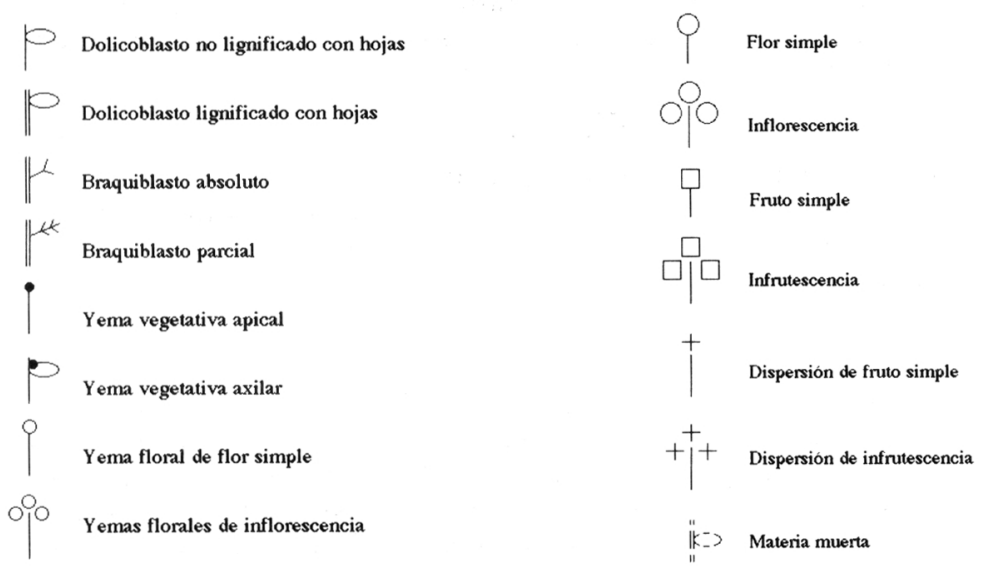

B. Fases fenológicas.
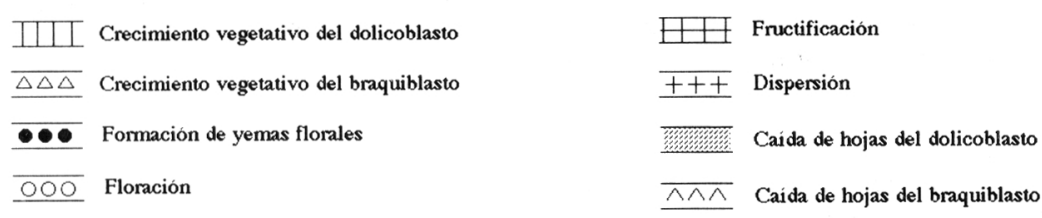


\section{RESULTADOS}

Como resultado del estudio realizado, presentamos las descripciones fenomorfológicas y los diagramas fenomorfológicos de las cinco especies tratadas .

\section{C. populifolius L. subsp. major (Pourret ex Dunal) Heywood}

Descripción fenomorfológica. Arbusto siempreverde de hasta 1.5 metros de altura. Nanofanerófito que se desprende de las ramas en sentido acrópeto. Hojas malacófilas de unos $70 \mathrm{~mm}$. de longitud y $40 \mathrm{~mm}$. de anchura. Presenta dos tipos de ramas: dolicoblastos que emergen ó desde los ápices de los dolicoblastos de la última estación ó desde viejos braquiblastos y braquiblastos que aparecen en las axilas de las hojas superiores de los dolicoblastos. Crecimiento vegetativo del dolicoblasto de Marzo a Agosto y del braquiblasto de Diciembre a Marzo. Prefloración de Abril a Mayo y floración en Mayo a Junio. Fructificación de Junio a Agosto y dispersión de Agosto a Noviembre. Caída de las hojas de los dolicoblastos en Junio y Agosto y de los braquiblastos de Febrero a Abril. Aparece en comunidades arbustivas y boscosas ombrófilas.

Diagrama fenomorfológico. Figura 2.

\section{C. ladanifer $\mathrm{L}$.}

Descripción fenomorfológica. Arbusto siempreverde de 1 a 2 metros de altura. Nanofanerófito que se desprende de las ramas en sentido acrópeto. Hojas malacófilas resinosas, de unos $70 \mathrm{~mm}$. de longitud y $15 \mathrm{~mm}$. de anchura. Presenta dos tipos de ramas: dolicoblastos que aparecen principalmente en los ápices de los braquiblastos ó en los ápices de los dolicoblastos de la última estación y braquiblastos parciales que se desarrollan en las axilas de las hojas de los dolicoblastos, parte de ellos crecen eventualmente en los nuevos dolicoblastos y los demás generalmente mueren. Crecimiento vegetativo del dolicoblasto de Mayo a Septiembre y del braquiblasto durante todo el año. Prefloración de Enero a Abril y floración en Abril-Mayo. Fructificación de Junio a Agosto y dispersión de Agosto a Diciembre. Caída de las hojas de los dolicoblastos de Junio a Agosto y de los braquiblastos durante todo el año. Constituyente principal de jarales, silicícola y heliófila.

Diagrama fenomorfológico. Figura 3.

\section{C. albidus $\mathrm{L}$.}

Descripción fenomorfológica. Arbusto siempreverde de hasta $1 \mathrm{~m}$. de altura. Nanofanerófito que se desprende de las hojas de modo acrópeto. Hojas malacófilas de unos $40 \mathrm{~mm}$. de longitud y $15 \mathrm{~mm}$. de anchura. Tiene dos tipos de ramas: dolicoblastos y braquiblastos parciales que se desarrollan en las axilas de las hojas de los dolicoblastos. Crecimiento vegetativo de los dolicoblastos de Abril a Agosto y de Diciembre a Febrero y de los braquiblastos durante todo el año. Prefloración de Marzo a Mayo y floración de Abril a Mayo. Fructificación de Mayo a Agosto y dispersión de Agosto a Diciembre. Caída de hojas de los dolicoblastos de Junio a Agosto y de los braquiblastos durante todo el año. Constituyente de matorrales heliófilos y xerófilos. Diagrama fenomorfológico. Figura 4.

\section{C. monspeliensis L.}

Descripción fenomorfológica. Arbusto siempreverde de hasta $1 \mathrm{~m}$. de altura. Nanofanerófito que se desprende de las ramas en sentido acrópeto. Hojas linear- 
lanceoladas, de unos $40 \mathrm{~mm}$. de longitud y $6 \mathrm{~mm}$. de anchura, malacófilas y resinosas. Presenta dos tipos de ramas: dolicoblastos y braquiblastos temporales que se desarrollan en las axilas de las hojas superiores del dolicoblasto. Crecimiento vegetativo del dolicoblasto de Abril a Noviembre y de los braquiblastos de Octubre a Abril. Prefloración y floración de Abril a Mayo. Fructificación de Mayo a Agosto y dispersión de Agosto a Noviembre. Caída de hojas del dolicoblasto de Abril a Agosto y de los braquiblastos en Junio y Julio. Aparece en matorrales y jarales degradados.

Diagrama fenomorfológico. Figura 5.

\section{C. salvifolius $\mathrm{L}$.}

Descripción fenomorfológica. Pequeño arbusto siempreverde de hasta $1 \mathrm{~m}$. de altura. Nanofanerófito que se desprende de las ramas de modo acrópeto. Hojas malacófilas de unos $20 \mathrm{~mm}$. de longitud y $10 \mathrm{~mm}$. de anchura. Presenta dos tipos de ramas: dolicoblastos que se desarrollan desde braquiblastos parciales o a veces desde yemas apicales de dolicoblastos de los últimos años y braquiblastos parciales temporales que se desarrollan en las axilas de las hojas de los dolicoblastos. La mayor parte de las inflorescencias son apicales aunque algunas se desarrollan desde yemas de ramas laterales que permanecían activas. Crecimiento vegetativo de los dolicoblastos de Mayo a Septiembre y de los braquiblastos de Septiembre a Junio. Prefloración de Febrero a Abril y floración de Abril a Junio. Fructificación de Junio a Septiembre y dispersión de Septiembre a Noviembre. Caída de las hojas del dolicoblasto de Junio a Octubre y de los braquiblastos de Mayo a Julio. Forma parte de aulagares y jarales ó en bosques algo degradados.

Diagrama fenomorfológico. Figura 6.

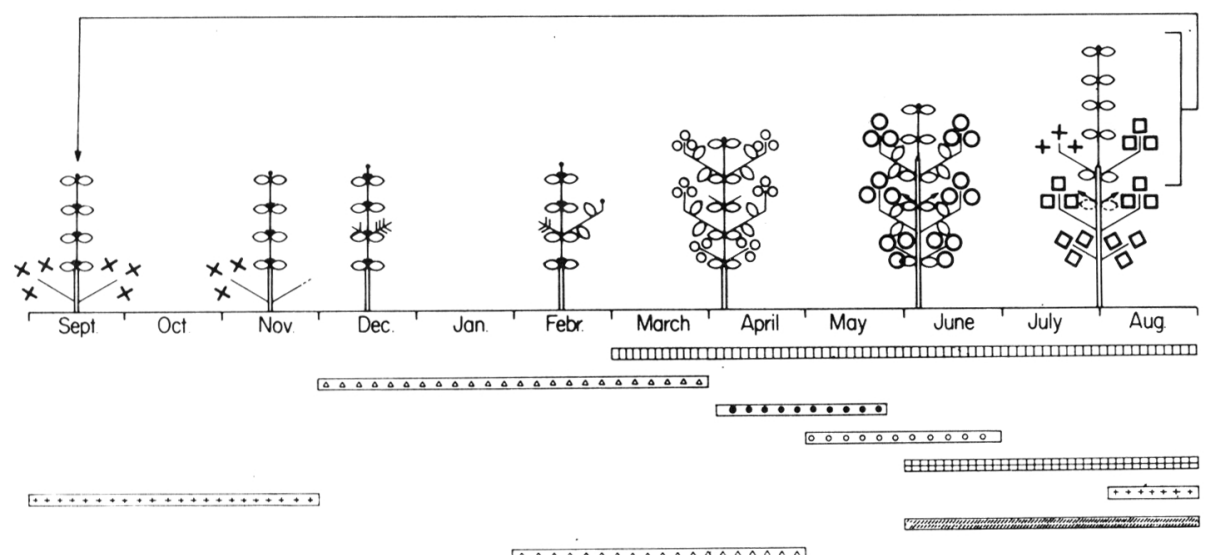

Figura 2. Diagrama fenomorfológico de C. populifolius L. subsp. major (Pourret ex Dunal) Heywood 


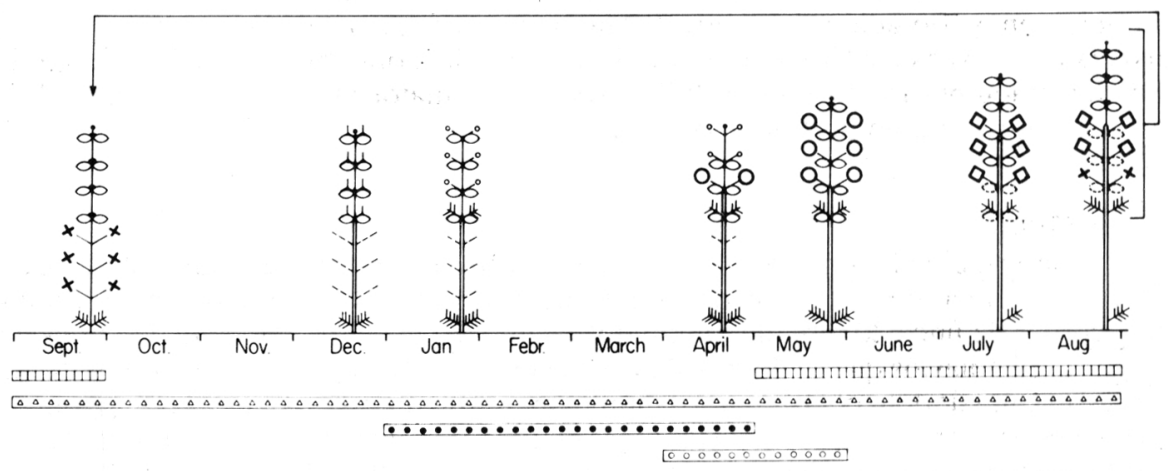

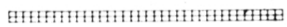

$\ldots+\ldots$

Figura 3. Diagrama fenomorfológico de C. ladanifer L.

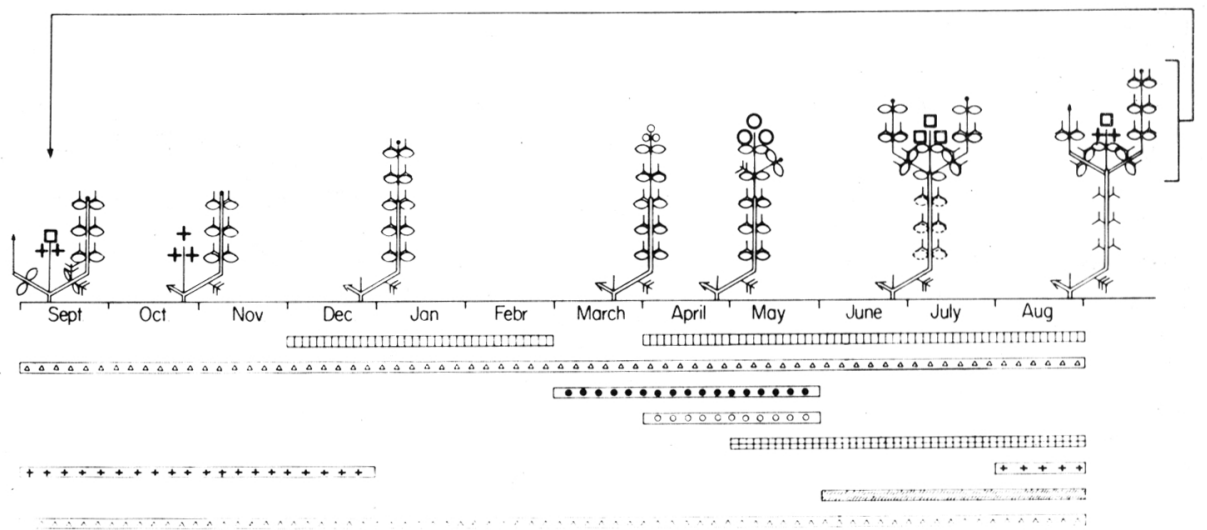

Figura 4. Diagrama fenomorfológico de C. albidus L. 


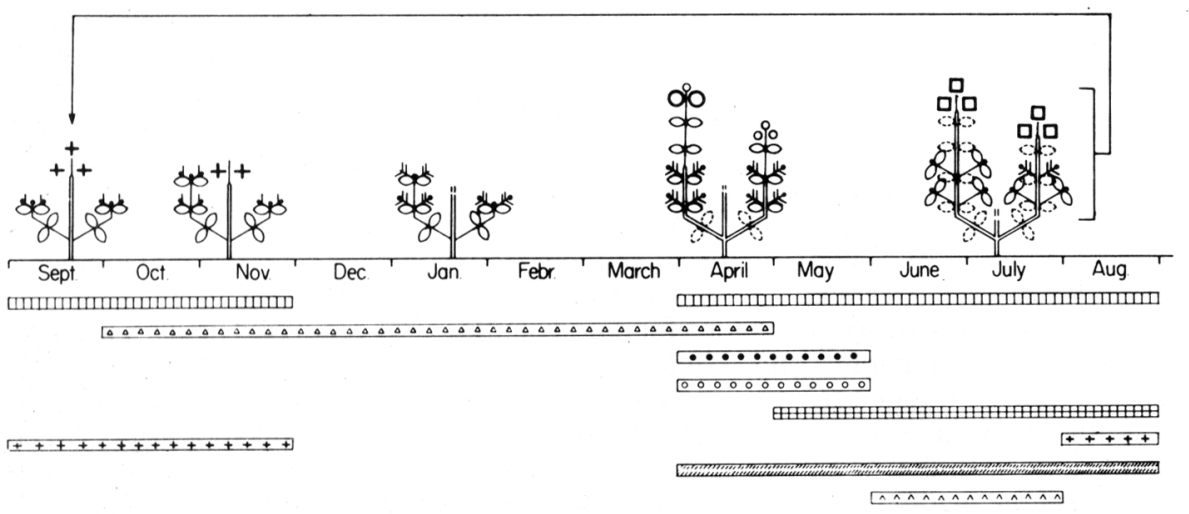

Figura 5. Diagrama fenomorfológico de C. monspeliensis L.

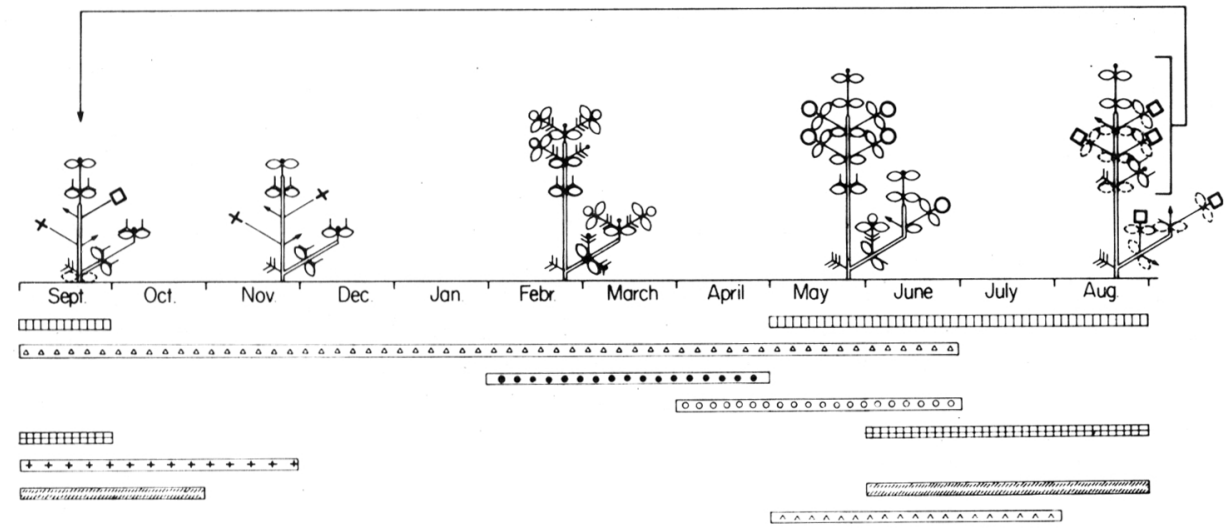

Figura 6. Diagrama fenomorfológico de C. salvifolius L. 


\section{CONCLUSIONES}

En función de los órganos de renovación de las cinco especies estudiadas, podemos observar que dos de ellas, $C$. monspeliensis y $C$. albidus, se caracterizan por presentar solo yemas vegetativas axilares; por el contrario $C$. populifolius, $C$. ladanifer y $C$. salvifolius presentan también yemas vegetativas apicales. Al primer grupo pertenece también C. villosus, especie estudiada en Israel (Orshan,1989) y al segundo grupo pertenece $C$. laurifolius, especie estudiada en Francia (Floret et al., 1989). El hecho de que la localización de las yemas de renovación sea solamente axilar o axilar y apical parece no estar relacionado con las áreas de distribución de las especies estudiadas o con su historia evolutiva, por lo que no podemos sugerir por el momento que esta característica tenga algún valor adaptativo.

En función de los resultados fenomorfológicos, las fenofases de las 5 especies estudiadas se comparan con los resultados obtenidos en Francia ( $C$. albidus y $C$. monspeliensis) e Israel (C. salvifolius) por Floret et al., (1989) y Orshan (1989). El crecimiento vegetativo de los dolicoblastos comienza en primavera tanto en las poblaciones estudiadas en este trabajo como en las francesas. Para las dos especies estrictamente mediterráneas occidentales ( $C$. populifolius y $C$. ladanifer) y para la de areal circunmediterráneo ( $C$. salvifolius) la duración de dicho crecimiento se prolonga hasta finales del verano. En Israel por el contrario el crecimiento de $C$. salvifolius se lleva a cabo durante casi todo el año con una parada en primavera. C. albidus crece en España (Málaga) y Francia durante todo el año con una parada en otoño. Para $C$. monspeliensis, especie de amplia distribución, el crecimiento en Málaga se prolonga hasta mediados de otoño, mientras que en las poblaciones francesas se realiza durante todo el año. Referente al crecimiento vegetativo de los braquiblastos, no se pueden establecer diferencias entre los formados en las axilas de las hojas de los jóvenes dolicoblastos, que elongarán posteriormente, y aquellos formados anteriormente y que no llegan a elongar. En $C$. ladanifer, $C$. albidus y $C$. salvifolius el crecimiento de los braquiblastos se realiza durante casi todo el año, mientras que en $C$. populifolius y $C$. monspeliensis ocurre en otoño e invierno. La floración de las 5 especies ocurre en primavera y/o principio del verano y la prefloración en invierno y primavera, no existiendo diferencias marcadas entre las poblaciones estudiadas y las francesas; por el contrario la prefloración y floración de $C$. salvifolius en Israel se adelanta un mes. La fructificación tiene lugar en Málaga durante la primavera y verano, y la dispersión en verano y otoño. Los datos para Francia no difieren marcadamente de los españoles, mientras que en Israel (C. salvifolius) ambas fenofases se realizan dos meses antes. La caída de hojas en los dolicoblastos tiene lugar en verano para las especies mediterráneas occidentales, y se adelanta algo en las especies circunmediterráneas, no existiendo diferencias marcadas entre las tres estaciones comparadas. En Israel la caída de hojas de los braquiblastos en $C$. salvifolius ocurre prácticamente durante todo el año, mientras que en España y Francia ocurre desde finales de primavera a principios de verano. En $C$. ladanifer y $C$. albidus la caída de hojas de los braquiblastos se realiza a lo largo de todo el año, por el contrario C. populifolius (invierno) y C. monspeliensis (verano) presentan una estacionalidad muy marcada.

\section{BIBLIOGRAFÍA}

ALJARO, M.E. \& G. MONTENEGRO -1981- Growth of dominant Chilean shrubs in the Andean Cordillera. Mountain research and development, 1: 287-291. 
DELEÓN LLAMAZARES, A. -1989-Caracterización Agroclimática de la Provincia de Málaga. M. A. P. A.

FLORET, C., M. J. GALAN, E. LE FLOC'H, F. LEPRINCE \& F. ROMANE. In: Orshan, G. (ed.). -1989- Plant pheno-morphological studies in Mediterranean type ecosistems. Kluwer Academic Publishers 404 pp.

GREUTER, W., H. M. BURDET \& G. LONG (eds.). -1984- Med-Checklist-1. Géneve.

HAGEMANN, I. - 1983- Wuchsformenuntersuchungen am Zentral-europaischen Hypericum. Arten. Flora, 173: 97-142.

HOFFMANN, A. -1972- Morphology and histology of Trevoa trinervis (Rhamnaceae) a drought deciduous shrub from the Chilean matorral. Flora, 161: 527-538.

HOFFMANN, A. J. -1981- Seasonal growth rhythm in Peumus boldus a deciduous tree of the Chilean mediterranean vegetation. Ecol. Plant., 2: 31-39.

HOFFMANN, A. J. \& A. E. HOFFMANN, -1976- Growth pattern and seasonal behaviour of the buds of Colliguaya odorifera, a shrub from the Chilean mediterranean vegetation. Can. J. Bot., 54: 1774-1787.

HOFFMANN, A. J. \& M. J. WALKER -1980-Growth habits and phenology of drought deciduous species in an altitudinal gradient. Can. J. Bot., 58: 1789-1796.

JOW, W. M., S. H. BULLOCK, \& J. KUMMEROW -1980- Leaf turnover rates of Adenostoma fasciculatum (Rosaceae). Amer. J. Bot., 67: 256-261.

KUMMEROW, J. - 1983- Comparative phenology of mediterranean type plants communities. In: Kruger, F. J., Mitchell D. T. and Jarvis J. V. M. (eds.) Mediterranean type ecosistems: the role of minerals. Springer N. Y. pp. 300-317.

LE ROUX, A., X. L. KYRIACOU, \& G. ORSHAN -1984- The phenomorphology of selected plants in mediterranean type ecosistems of South Africa. Bull. Soc. Bot. Fr., 131: 441-450.

LEPRINCE, P. -1987-Cycles pheno-morphologiques et forms de croissance de quelques especes du Provence. Adaptation a l'environment méditerraneen. Thèse Docteur de $3^{\mathrm{eme}}$ cycle en ecologie. Université de droit, d'ecconomie et des Sciences D'Aix-Marseille. 170 pp.

MONTENEGRO, G., M. E. ALJARO, \& J. KUMMEROW -1979- Growth dynamics of Chilean matorral shrubs. Bot. Gaz., 140: 114-119.

MONTENEGRO, G., A. J., HOFFMANN, M.E. ALJARO \& A. E. HOFFMANN -1979- Satureja gilliesii a poikilohydric shrub from Chilean mediterranean vegetation. Amer.J.Bot., 57: 12061213.

NIETO CALDERA, J. M., A. V. PÉREZ LATORRE y CABEZUDO, B. -1991- Biogeografía y Series de Vegetación de la provincia de Málaga (España). Acta Bot. Malacitana, 16(2): 417436.

ORSHAN, G. In: Orshan, G. (ed.). -1989-Plant pheno-morphological studies in Mediterranean type ecosistems. Kluwer Academic Publishers $404 \mathrm{pp}$.

ORSHAN, G. -1964- Seasonal dimorphism of desert and mediterranean chamaephytes and their significance as a factor in their water economy. In: Rutter, A. J. and Whitehead F. H. (eds.) Water in relation to plants. Blackwell, Oxford, pp. 206-222.

RIVAS MARTÍNEZ, S. -1987- Memoria del Mapa de las series de Vegetación de España. M. A. P. A.-I. C. O. N. A.

RUTHERFORD, C. M. \& M. D. PANGOS -1982- Seasonal woody plant shoot growth in Burkea africana - Ochna pulchra savana. South Afr. J. Bot., 1: 104-116.

WARBURG, E. F. -1968- Cistus L. in Tutin et al (eds.). Flora Europaea. Cambridge.

WESTMAN, W. E. -1981- Seasonal dimorphism of foliage in Californian Coastal sage scrub. Oecologia, (Berlin) 51:385-388.

(Aceptado para su publicación en Junio de 1992)

Dirección de los autores. B. Cabezudo, A. V. Pérez Latorre, T. Navarro y J. M. Nieto: Departamento de Biología Vegetal. Universidad de Málaga. España. G. Orshan: Botany Department. The Hebrew University of Jerusalem. Israel. 